\title{
Unabhängig von Überdosen mehr Todesfälle
}

Fragestellung: Erhöht die Behandlung von nicht tumorbedingten Schmerzen mit retardierten Opioiden die Mortalität?

Hintergrund: Insbesondere im angloamerikanischen Raum sind in den vergangenen Jahren die Verordnungen von Opioiden beim Nichttumorschmerz dramatisch gestiegen. Die Behandlung mit Opioiden ist mit einer dosisabhängig erhöhten Mortalität durch Opioidüberdosierungen assoziiert. Diese Untersuchung ging der Frage nach, wie sich eine Behandlung mit Opioiden im Vergleich zu einer Schmerztherapie mit analgetischen Antidepressiva oder Antikonvulsiva auf die Gesamtmortalität auswirkt.

Patienten und Methodik: Es handelt sich um eine retrospektive Kohortenstudie aus den USA, in der die Verläufe von 23.308 Neuverschreibungen von retardierten Opioiden und 131.883 Neuverschreibungen von Antidepressiva/Antikonvulsiva zur Behandlung von moderaten oder starken Nichttumorschmerzen berücksichtigt wurden. Die Gesamt- und ursachenspezifische Mortalität der Kohorte wurde anhand der Diagnosen in den Sterbeurkunden ausgewertet. Um einen möglichen Bias durch andere Faktoren zu reduzieren, wurden aus diesen Fällen zwei Gruppen von jeweils 22.912 Verschreibungen für Opioide beziehungsweise die Kontrollmedikation extrahiert, die für insgesamt 122 Kovariaten mit vermutetem Einfluss auf die

Wayne AR, Chung CP, Murray KT et al. Prescription of longacting opioids and mortality in patients with chronic noncancer pain. JAMA 2016; 315: 2415-23 Gesamtmortalität (z.B. demografische Variablen, Schmerzdiagnosen, psychiatrische Diagnosen, kardiovaskuläre Risikofaktoren,
Komedikation etc.) gematcht waren. Patienten über 75 Jahre, hospitalisierte Patienten und Patienten in einem palliativen Setting wurden von der Analyse ausgeschlossen.

Ergebnisse: Die mittlere Dosis der Opioidgruppe betrug $50 \mathrm{mg}$ Morphinäquivalent, die der Kontrollgruppe $600 \mathrm{mg}$ Äquivalent zu Gabapentin beziehungsweise $25 \mathrm{mg}$ Amitriptylin. Der durchschnittliche Follow-up der Opioidgruppe (mittleres Alter 48 Jahre, 60\% Frauen) betrug 176 Tage, in dieser Zeit traten 185 Todesfälle auf. Der durchschnittliche Follow-up der Kontrollgruppe, die mit analgetischen Antidepressiva oder Antikonvulsiva behandelt wurde, betrug 128 Tage mit 87 Todesfällen. Die Auftretenswahrscheinlichkeit von Todesfällen insgesamt war in der Opioidgruppe signifkant höher (Hazard Ratio [HR] Gesamtmortalität 1,64). Diese war bedingt durch eine erhöhte Rate von "out-of-hospital deaths" (HR 1,9). Hier zeigte sich nicht nur ein erhöhtes Auftreten von Todesfällen durch akzidentelle Überdosierung (HR 3,37), sondern auch ein erhöhtes Auftreten von kardiovaskulären Todesfällen (HR 1,65) im Vergleich mit einer Behandlung mit Antidepressiva oder Antikonvulsiva. Die Wahrscheinlichkeit für Todesfälle in der Opioidgruppe war insbesondere in den ersten 30 Tagen der Therapie erhöht und abhängig von der Opioiddosierung.

Schlussfolgerungen: Die Behandlung von nicht tumorbedingten Schmerzen mit Opioiden ist mit einer höheren Gesamtmortalität verbunden als eine Behandlung mit Antikonvulsiva oder Antidepressiva. Diese ist nicht nur auf eine erhöhte Rate an Todesfällen durch akzidentelle Überdosierung zurückzuführen. Vielmehr erhöht eine Opioidtherapie auch das Auftreten von kardiovaskulär bedingten Todesfällen.

\section{Fortführung der Opioidtherapie kritisch und regelmäßig überprüfen}

Diese methodisch aufwändige, gut kontrollierte Studie an einem sehr großen Patientenkollektiv zeigt, dass eine Behandlung mit retardierten Opioiden mit einer erhöhten Mortalität verbunden ist. Diese betrifft auch das Risiko für

Weitere Infos auf springermedizin.de

Opioide bei Nichttumorschmerz im höheren Lebensalter

In diesem Artikel sind Indikationen, Besonderheiten und

Risiken einer Opioidtherapie bei Nichttumorschmerz im höheren Lebensalter zusammengefasst.

Diesen Artikel finden Sie, indem Sie den Titel in die Suche eingeben. kardiovaskuläre Todesfälle. In Zusammenschau mit den aktuellen Metaanalysen zur Wirksamkeit von Opioiden beim Nichttumorschmerz unterstreicht diese Studie, dass die Indikation und Fortführung einer Opioidtherapie beim Nichttumorschmerz kritisch und regelmäßig geprüft werden sollte.

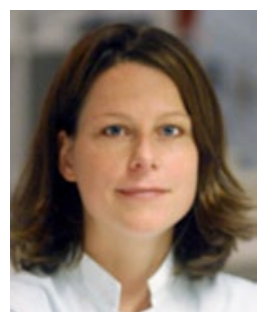

Prof. Dr. med. Ulrike Bingel, Essen

Professur für Klinische Neurowissenschaften, Leitung der Schmerzambulanz, Klinik für Neurologie der Universitätsklinik Essen E-Mail: ulrike.bingel@uk-essen.de 